\title{
A Study and Evaluation on Route Guidance of a Car Navigation System Based on Augmented Reality
}

\author{
Kengo Akaho ${ }^{1}$, Takashi Nakagawa ${ }^{1}$, Yoshihisa Yamaguchi ${ }^{1}$, \\ Katsuya Kawai ${ }^{1}$, Hirokazu Kato ${ }^{2}$, and Shogo Nishida ${ }^{3}$ \\ ${ }^{1}$ Advanced Technology R\&D Center, Mitsubishi Electric Corporation, \\ 8-1-1, Tsukaguchi-honnmachi, Amagasaki, Hyogo, 661-8661, Japan \\ \{Akaho.Kengo@eb, Nakagawa.Takashi@dy, Yamaguchi.Yoshihisa@dp, \\ Kawai.Katsuya@dn\}.MitsubishiElectric.co.jp \\ ${ }^{2}$ Graduate School of Information Science, Nara Institute of Science and Technology \\ kato@is.naist.jp \\ ${ }^{3}$ Graduate School of Engineering Science, Osaka University, \\ 1-3, Machikaneyama, Toyonaka, Osaka, 560-8531, Japan \\ nishidalsys.es.osaka-u.ac.jp
}

\begin{abstract}
We have developed AR-Navi, which is a car navigation system based on augmented reality technology. AR-Navi overlays computer graphics element on live video feed captured by the in-vehicle camera. In this study, we investigated and evaluated information presentation methods in intersection guidance by AR-Navi, from consideration of ease of understanding and also safety. As a result of the evaluations, we confirmed ease of understanding, safety, and the characteristics of AR-Navi in comparison with CG-Navi.
\end{abstract}

Keywords: Car navigation systems, Augmented reality, Route guidance.

\section{Introduction}

At present, car navigation systems play a prominent part in the safety and smoothing of road traffic [1]. Many of today's car navigation systems involve route guidance that mainly uses computer graphics (CG). Car navigation systems that use CG are called CG-Navi in this paper. However, CG-Navi cannot completely reproduce the real world that the user sees through the windshield. For that reason, it is difficult for the user to accurately and quickly recognize small differences in location, so the user can get lost or be unable to drive safely because he is peering at the car navigation screen.

Thus, to increase the ease of understanding and safety of car navigation systems, systems have been proposed for providing guidance at intersections, which use an invehicle camera to capture live video footage in real time from in front of the vehicle, then overlay CG elements that have been created by using augmented reality technology onto that live video feed.

We are currently developing a car navigation system based on augmented reality technology. The car navigation system we are developing is called AR-Navi [2]. 
To implement this AR-Navi, we must resolve the following two issues:

1. Improved precision of the matching of the live video feed and CG elements

2. Presentation of information about intersection guidance, from consideration of ease of understanding and also safety while driving

To tackle the first issue, we have improved the precision with which the live video feed and CG are overlaid [3].

In this paper, we describe means of tackling the second issue in AR-Navi. We define guidance that enables the user to accurately comprehend easy-to-understand information within a short time and also without error, and investigate a method of presenting AR-Navi information that implements that guidance. In addition, we are studying guidance that does not draw the user's attention away for long, to ensure that the user avoids gazing at the car navigation screen while driving, from consideration of safety. In order to implement those objectives, the following two problems must be resolved:

1. Since AR-Navi guidance is based on various different kinds of information being displayed live, the amount of information on screen tends to become large (*).

* In this paper, a car navigation screen that causes the user to take a long time between looking at the screen and understanding the information on that screen is defined to have a large amount of information.

2. Since the video feed captured by the in-vehicle camera consists of moving images at $30 \mathrm{fps}$, the user's eyes will tend to follow the movements in the video when it is displayed on the car navigation screen, and there is a danger that he will gaze fixedly at the screen.

To address the first problem, we have investigated designs for the display of intersection guidance that are easy to understand with a small amount of information. To address the second problem, we have proposed a "best shot" display method as guidance that does not use moving images. In addition, we have implemented a prototype system that incorporates these designs and have conducted driving evaluation experiments on public roads to evaluate the ease of understanding and safety of AR-Navi.

In this paper, we describe the above method and evaluation experiments, then discuss the results of the evaluation experiments.

\section{Related Research}

We shall now introduce some research into car navigation systems that use augmented reality technology.

Ko et al. have proposed a method of positioning by cross-checking three types of data (road video footage that is input from a CCD camera, the current position of the vehicle, and a road configuration model created from road map data), and have implemented highly precise overlaying of a live video feed and CG[4].

Sawano et al. describes how they showed test subjects some simulated video footage for intersection guidance, which was generated by using augmented reality technology, and guidance displayed by conventional CG-Navi. From comparisons by questionnaire, they found that the display of generated video footage was easier to 
understand [5]. The research done by Sawano et al. evaluated the ease of understanding of information presented by car navigation systems that used augmented reality technology, which is the same as in our research. They implemented experiments in an environment in which users could gaze at a screen, but there was insufficient investigation in situations in which car navigation system is used while driving.

\section{Methods of Presenting Information in Intersection Guidance}

\subsection{Design of Intersection Guidance Display}

To ensure that the user does not gaze fixedly at the screen, the car navigation systems display design must provide display contents that has a reduced amount of information but which enables the user to recognize useful information on the route guidance as simply as possible. We have determined that types of guidance that have any of the following characteristics have a large amount of information:

- Guidance with lots of text and CG

- Guidance using complicated diagrams

- Guidance in which displayed CG elements change dynamically

It is considered that if we could implement a display design which does not have any of the above characteristics and which traces the route by lines and arrows, turn locations could be conveyed intuitively to the user with a small amount of information. Thus we propose the following two types of guidance that focus on the locations at which $\mathrm{CG}$ elements are displayed to indicate the route:

1. On-road route display method

2. Overhead arrow display method

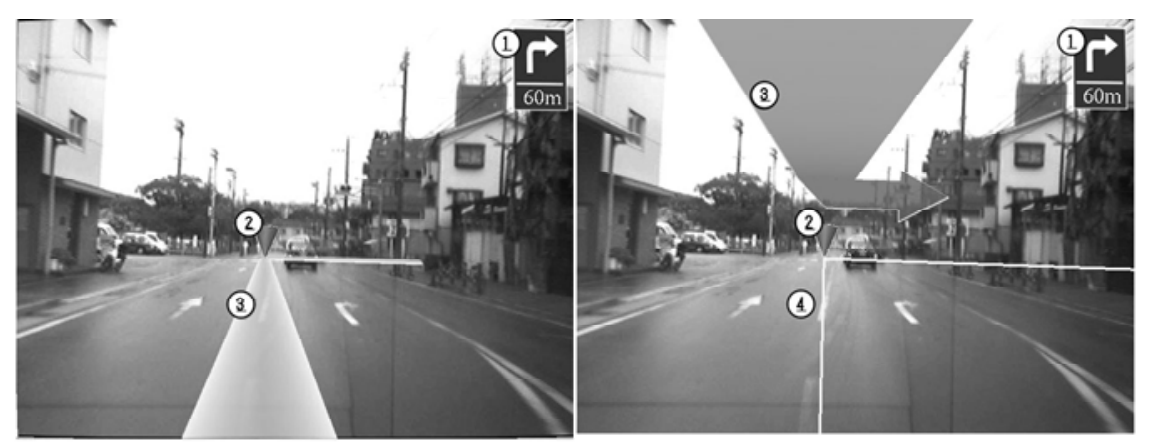

1. The distance to Intersection, turning direction

2. Blue symbol indicating the center of Intersection

3. Yellow route or overhead arrow indicating the navigated route

4. Yellow line indicating the navigated route

Fig. 1. On-road route guidance and overhead route guidance 
Since the route is displayed on the ground with display design 1, the correspondence with the road is easy to see, but if there are any vehicles ahead, the CG elements are displayed on those vehicles, which give the users an uncomfortable feeling. Since the route is displayed overhead with display design 2 , this does not affect any vehicles ahead and there is also little shielding, but it is thought that it will be difficult to see the correspondence with the road quickly, in comparison with display design 1. Each of these designs was implemented as AR-Navi.

An example of the guidance provided by the on-road route display method and the overhead arrow display method are shown in Fig. 1. These examples are just single examples, and it is possible to replace the cone that indicates the intersection center with an arrow and make changes such as displaying side roads.

\subsection{Best Shot Display Method}

As shown in Section 1, it is dangerous to display moving images on a car navigation screen. Guidelines for the safe handling of image display devices, which have been set out by the Japan Automobile Manufacturers Association, Inc., also state that moving images should not be displayed on such devices [6].

That is why it has been thought that, if the setup is such that the screen is not updated frequently while the user is looking at the car navigation screen, the user will not gaze fixedly at the screen. To that end, we propose a lengthened update period for the moving images, to display single frames at a time. If the moving images are simply displayed as single frames, the generation of adverse conditions such as backlighting could cause the display of an image that is unsuitable for use in the guidance for a fixed time. We therefore propose a best shot display method by which an image that is suitable for the guidance is selected and displayed from the captured moving images.

To implement this method, we study the fundamentals of images that are unsuitable for guidance. When an image captured from an in-vehicle camera is unsuitable, that image is not selected at the "best shot". In this paper, the following conditions cause images to be unsuitable:

1. The image is too dark or bright

2. The user's vehicle is not following the line of the road

3. The user's vehicle and the target intersection of the guidance are too close

Since it is considered that nearer images within the video footage that the user is currently seeing are easier to understand, the best shot display method selects the latest image as the best shot, from among the moving images captured by the invehicle camera but excluding unsuitable images.

\section{Evaluation Experiments}

We created a prototype AR-Navi system by implementing the best shot display method we propose in this paper and intersection guidance display designs, and installed it in a vehicle by mounting it as one function of the car navigation systems. We evaluated the ease of understanding and safety of this AR-Navi, and performed evaluation experiments while driving on public roads to compare conventional CGNavi and AR-Navi, with the objective of clarifying its characteristics. 


\subsection{Preliminary Experiments}

We performed pilot driving tests on public roads while using AR-Navi, as preliminary experiments. We adjusted the parameters of two items during the runs: the display design and the image update timing spacing of the best shot display method. Since the settings shown in Table 1 were determined to be easy to understand, we implemented the experiments with those settings.

Table 1. Configuration of our system

\begin{tabular}{ll}
\hline Setting Items & Configuration \\
\hline Display Design & On-road route display method \\
Image update timing spacing & 0.75 seconds \\
\hline
\end{tabular}

\subsection{Details of this Experiment}

Experimental conditions. The experimental conditions relating to details are listed in Table 2. All test subjects drive on a daily basis and have experience of using car navigation systems.

Table 2. Configuration of experiment

\begin{tabular}{ll}
\hline $\begin{array}{l}\text { Experimental } \\
\text { conditions }\end{array}$ & Configuration \\
\hline Test subjects & Three males aged 29 to 32. \\
Time required & Approximately 50 minutes for each test subject \\
experiments & for one display design \\
Time and weather & Between 11 AM and 4 PM, in both sunny and cloudy weather \\
$\begin{array}{l}\text { Experiment course } \\
\text { Comparison }\end{array}$ & Residential areas, ordinary roads with one or two lanes each way \\
guidance types & Total of three types: CG-Navi and versions 1 and 2 of AR-Navi \\
\hline
\end{tabular}

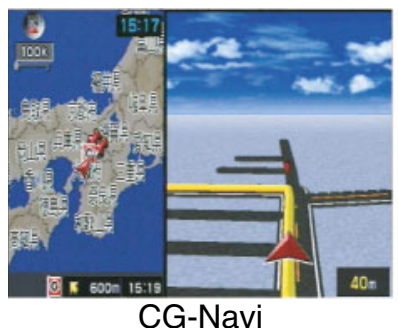

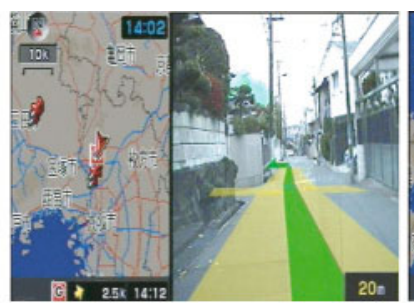

AR-Navi Ver.1

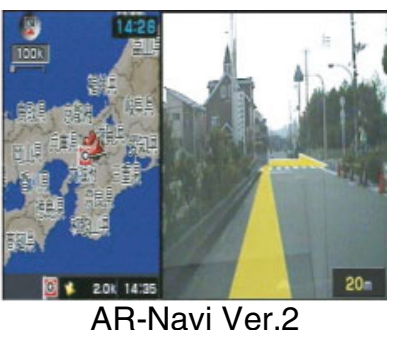

AR-Navi Ver.2

Fig. 2. Examples of car navigation guidance

The display designs used in these experiments are shown in Fig. 2. In these experiments, we used CG-Navi and two versions of AR-Navi with the on-road route display method that was decided upon in the preliminary experiments of Section 4.1. In this paper, these designs are called CG-Navi, AR-Navi Ver. 1, and AR-Navi Ver. 
2, respectively. With the display design of AR-Navi Ver. 1, side roads other than the route that the user's vehicle travels on were shown, in addition to the route leading to our company. With the display design of AR-Navi Ver. 2, only the route that the user's vehicle travels on was shown, and side roads were not displayed. AR-Navi Ver. 1 is a display design with a larger amount of data than AR-Navi Ver. 2. To investigate the effects of differences in the amount of information in the display design on safety and ease of understanding, we used two display designs.

Experimental method. The test subjects were given tasks while riding in a vehicle traveling on public roads. Each test subject determined the position and direction to turn from the car navigation guidance, and specified the driving operations. He specified the turn location and direction at the point at which he was confident about the turn location.

Each test subject performed the tasks from the passenger seat, and was instructed to check the forward direction as if intending to drive. In addition, the test subjects were permitted to operate a button that can turn the car navigation screen on or off, to ensure they are not conscious of the screen more than necessary, by turning the display on only when looking at the car navigation screen. The test subjects were also allowed to familiarize themselves with the tasks by performing familiarization runs beforehand.

Evaluation method. We examined the following four items, and also investigated the characteristics of AR-Navi :

1. The results relating to correct/false identification of intersections by the car navigation systems.

2. The visual-contact time ratio for car navigation systems (the total car navigation visual-contact time at a certain intersection, divided by the total time from the start of guidance to that intersection until the vehicle passes the intersection) and the visual-contact time per look

3. Distances from the intersection when the user gives the response when he completely understands the turn location

4. Subjective opinions of test subjects, collected from questionnaires

To study these evaluation items, we recorded each test subject's face, the front of the vehicle, and the car navigation screen as video images, and also measured the times the button was pressed.

\subsection{Results and Discussion}

First of all, the results relating to correct/false identification of intersections are shown in Table 3 and intersection information generated by false recognitions is shown in Table 4. Since there were few differences in the numbers of false recognitions, we could confirm that there were no major differences between the guidance types in the overall ease of understanding.

We will now discuss the results relating to visual-contact time. The visual-contact time ratio at each intersection when the test subjects were looking at the car navigation screen are shown in Fig. 3 and data relating to single-look visual-contact times is shown in Fig. 4. 
Table 3. Numbers of false recognitions of turn locations

\begin{tabular}{lll}
\hline & Number of Intersections & Number of falsely recognized intersections \\
\hline CG-Navi & 71 & 2 \\
AR-Navi Ver. 1 & 96 & 3 \\
AR-Navi Ver. 2 & 91 & 2 \\
\hline
\end{tabular}

Table 4. Information on intersections that generated false recognitions

\begin{tabular}{|c|c|c|}
\hline $\begin{array}{l}\text { Intersection } \\
\text { No. }\end{array}$ & Guidance Type & Characteristics \\
\hline 1 & CG-Navi & Intersection of a strange shape \\
\hline 2 & CG-Navi & $\begin{array}{l}\text { Intersection where the location of the intended turn is a } \\
\text { narrow road that is difficult to find. }\end{array}$ \\
\hline 3 & AR-Navi Ver. 1 & Intersection with a strange configuration \\
\hline 4 & AR-Navi Ver. 1 & The road leading to the intersection to turn into is curved. \\
\hline 5 & AR-Navi Ver. 1 & Within a narrow residential street, with many side roads. \\
\hline 6 & AR-Navi Ver. 2 & The road to turn into in the guidance was extremely narrow. \\
\hline 7 & AR-Navi Ver. 2 & Intersection located on uphill slope. \\
\hline
\end{tabular}

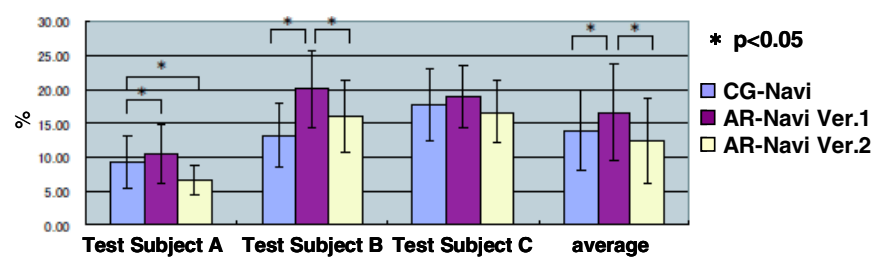

Fig. 3. Average visual-contact time ratios

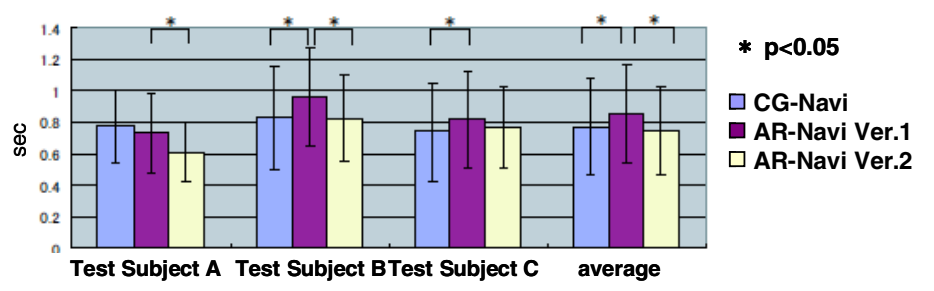

Fig. 4. Average single-look visual-contact times

As the result of performing t-tests at the 5\% significance level on averages, we found no significant differences between CG-Navi and AR-Navi Ver. 2 in visual-contact time ratio and single-look visual-contact time when looking at the car navigation screen. The single-look visual-contact time and visual-contact time ratio for AR-Navi Ver. 1 were somewhat higher than for CG-Navi and AR-Navi Ver. 2, so there was a significant difference.

These results are thought to be due to the increased visual-contact time caused by the larger amount of information displayed by AR-Navi Ver. 1, such as side roads. 
However, since the single-look visual-contact time average was no more that one second even with AR-Navi Ver. 1, this is thought to be no great problem from the safety viewpoint. In addition, we were able to confirm that there was not great difference in ease of understanding between AR-Navi and CG-Navi.

Results relating to distances from intersection when turn locations are specified are shown in Fig. 5. From the results of t-tests performed between the different types of guidance, significant differences were seen only between AR-Navi Ver. 1 and ARNavi Ver. 2. These were thought to be due to the lack of information on side roads in AR-Navi Ver. 2. Since there is no information on side roads in AR-Navi Ver. 2, this is thought to be why the subjects were not confident about the turn location until close to the intersection.

As a result, we confirmed that both CG-Navi and AR-Navi enabled confidence in the turn location at about the same distance from the intersection, by displaying side road information. We also confirmed that the effects when there is no side road information have large individual differences.

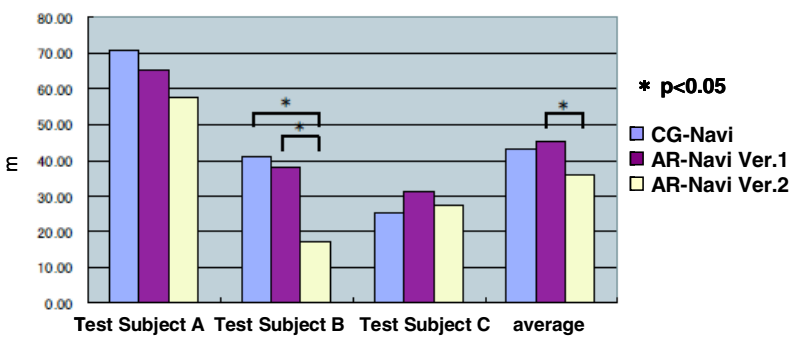

Fig. 5. Distance from intersection at specification

Table 5. Questionnaire results

\begin{tabular}{lllll}
\hline & $\begin{array}{l}\text { Distance from } \\
\text { intersection }\end{array}$ & $\begin{array}{l}\text { Time required } \\
\text { recognition }\end{array}$ & $\begin{array}{l}\text { Confidence } \\
\text { at time of turn }\end{array}$ & $\begin{array}{l}\text { Overall } \\
\text { evaluation }\end{array}$ \\
\hline CG-Navi & 5.00 & 4.67 & 5.00 & 4.67 \\
AR-Navi Ver. 1 & 4.67 & 4.33 & 6.33 & 5.00 \\
AR-Navi Ver. 2 & 4.67 & 4.67 & 6.00 & 4.67 \\
\hline
\end{tabular}

The results of questionnaires that the test subjects answered after the evaluation experiments are shown in Table 5. The items covered by the questionnaire were averaged to give questionnaire results for all the test subjects. We asked the test subjects to evaluate each item of the questionnaire in seven steps, with 1 being the worst evaluation and 7 being the best.

From the questionnaire results and open-response questions, we were able to confirm that the overall ease of understanding with AR-Navi was on the same order as that with CG-Navi, and that the guidance was more difficult to understand at some distance from each intersection but because easy to understand closer to the intersection, in comparison with CG-Navi. In addition, the open-response questions showed that all of the test subjects felt there was substantially no difference between AR-Navi Ver. 1 and AR-Navi Ver. 2. 
From the results of the evaluation experiments described in this Section, we confirmed that the ease of understanding during rapid visual-contact was about the same for CG-Navi and AR-Navi, when viewed overall. Regarding the safety of AR-Navi, we confirmed that it can be adjusted to about the same as that of CG-Navi, by controlling the amount of information in the display design. We also confirmed that the characteristics of AR-Navi in comparison with CG-Navi are such that the guidance is difficult to understand when far from an intersection but becomes even easier to understand when close to the intersection. In addition, since only a small number of experiments within these experiments related to intersections that generated false recognitions, we performed follow-up experiments relating to the intersections that generated those false recognitions to enable a detailed analysis. We performed follow-up experiments in a desktop environment using the display of a PC. In these follow-up experiments, we have confirmed that AR-Navi is easier than CG-Navi to understand when the road to turn into is narrow and difficult to find, and at intersections where there are many side roads. On the other hand, we also confirmed that AR-Navi is more difficult to understand than CG-Navi with intersections of strange configuration or which have roads proceeding in oblique directions.

\section{AR-Navi Improvement Suggestions}

From the results of these evaluation experiments, we were able to confirm the characteristics of CG-Navi and AR-Navi, as shown in Table 6.

As a method of resolving the problem that the configuration of an intersection is difficult to understand, we could consider using AR-Navi only when close to the intersection. AR-Navi is easy to understand for giving clear guidance of the turn location close to the intersection, and it also has little misalignment due to vibration of the vehicle and the intersection is not shielded much by buildings or the like.

In addition, the scale of the map displayed on the left side of the screen was made small but in practice this map can be used to help recognize the configuration of the intersection. For that reason, it is thought that this will reduce the problem that the configurations of some intersections are difficult to understand with AR-Navi.

Table 6. Characteristics of each car navigation system type

\begin{tabular}{|c|c|c|c|c|}
\hline & $\begin{array}{l}\text { When } \\
\text { distant from } \\
\text { intersection }\end{array}$ & $\begin{array}{l}\text { Directly } \\
\text { before intersection }\end{array}$ & $\begin{array}{l}\text { When } \\
\text { intersection } \\
\text { is shielded }\end{array}$ & $\begin{array}{l}\text { Multiple roads } \\
\text { and complicated } \\
\text { narrow alleyways }\end{array}$ \\
\hline $\begin{array}{l}\text { CG- } \\
\text { Navi }\end{array}$ & $\begin{array}{l}\text { Easy to } \\
\text { understand }\end{array}$ & $\begin{array}{l}\text { Difficult to understand } \\
\text { the specific location }\end{array}$ & $\begin{array}{l}\text { Easy to } \\
\text { understand }\end{array}$ & $\begin{array}{l}\text { Difficult to determine } \\
\text { the specific location }\end{array}$ \\
\hline $\begin{array}{l}\text { AR- } \\
\text { Navi }\end{array}$ & $\begin{array}{l}\text { Difficult to } \\
\text { understand }\end{array}$ & $\begin{array}{l}\text { Easy to understand the } \\
\text { specific location }\end{array}$ & $\begin{array}{l}\text { Difficult to } \\
\text { understand }\end{array}$ & $\begin{array}{l}\text { Easy to identify } \\
\text { other roads. }\end{array}$ \\
\hline
\end{tabular}

\section{Conclusions}

In this paper, we investigated and evaluated information presentation methods of car navigation systems in intersection guidance by AR-Navi, from consideration of ease of understanding and also safety. 
As a result of the evaluations, we confirmed that ease of understanding AR-Navi is similar between to that of CG-Navi when viewed overall. Furthermore, we confirmed that the safety of AR-Navi would cause no problems if the amount of information displayed is adjusted to be restricted to about the amount of information displayed by AR-Navi Ver. 2. We have also clarified the characteristics of AR-Navi in comparison with CG-Navi.

\section{References}

1. Japanese Ministry of Land, Infrastructure, Transport and Tourism ITS HomePage, http: / /www.mlit.go.jp/road/ITS/j-html/

2. Yamaguchi, Y., Nakagawa, T., Akaho, K., Honda, M., Kato, H., Nishida, S.: AR-Navi: an in-vehicle navigation system using video-based augmented reality technology. In: Proceedings of the 12th International Conference on Human-Computer Interaction, pp. 1139--1147 (2007)

3. Yamaguchi, Y., Nakagawa, T., Akaho, K., Honda, M., Kato, H., Nishida, S.: Evaluation for Adjustment Method of Vehicle's Location by Recognizing Crosswalks. In: Proceedings of the 13th International Conference on Human-Computer Interaction, pp. 648-656 (2009)

4. Hu, Z., Uchida, K.: Solution of Camera Registration Problem via 3D-2D Parameterized Model Matching for On-Road Navigation. International Journal of Image and Graphics 4(1), 3-20 (2004)

5. Sawano, H., Okada, M.: Real-time Video Processing by a Car-mounted Camera and Its Application to Car Navigation Systems by an Augmented Reality-based Display Method. The Journal of the Society for Art and Science 5(2), 57-68 (2006)

6. Japan Automobile Manufacturers Association: Guideline for In-vehicle Display Systems Version 3.0 (2004), http: / /www. jama.or.jp/safe/guideline/index.html 